\title{
ACREDITACIÓN EN SECTOR SALUD - SOCIEDAD INTERNACIONAL PARA LA CALIDAD EN EL CUIDADO DE LA SALUD (INTERNATIONAL SOCIETY FOR QUALITY IN HEALTH CARE - ISQUA)
}

\author{
Lyda Marcela Vargas Pérez \\ Jessica Alejandra Báez Agudelo
}

Monografía para optar al título de Auditora de Salud

Universidad Santo Tomás

Especialización en Auditoría de Salud

Bogotá

2018 


\title{
ACREDITACIÓN EN SECTOR SALUD - SOCIEDAD INTERNACIONAL PARA LA CALIDAD EN EL CUIDADO DE LA SALUD (INTERNATIONAL SOCIETY FOR QUALITY IN HEALTH CARE - ISQUA)
}

\author{
Lyda Marcela Vargas Pérez \\ Jessica Alejandra Báez Agudelo
}

Monografía para optar al título de Auditora de Salud

\begin{abstract}
Asesora
Alejandra Valenzuela Cazés

Fisioterapeuta

Esp. en Docencia Universitaria
\end{abstract}

Mg. en Salud Pública

Universidad Santo Tomás

Especialización en Auditoría de Salud

Bogotá

2018 


\section{TABLA DE CONTENIDO}

1. PROBLEMA DE INVESTIGACIÓN............................................................................ 4

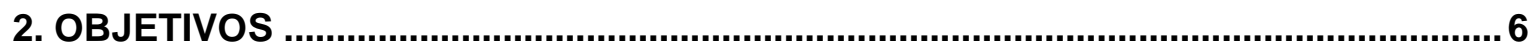

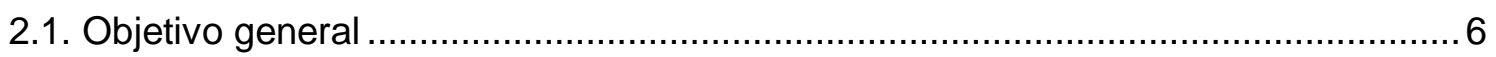

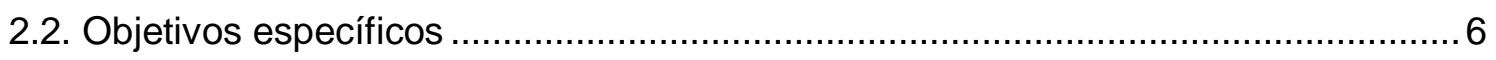

3. JUSTIFICACIÓN ................................................................................................ 7

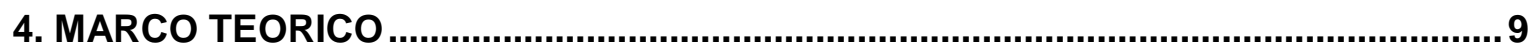

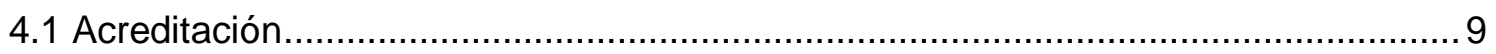

4.2 Internacional Society for Quality in Health Care (ISQua) ................................... 10

4.3. Principios de ISQua .............................................................................. 10

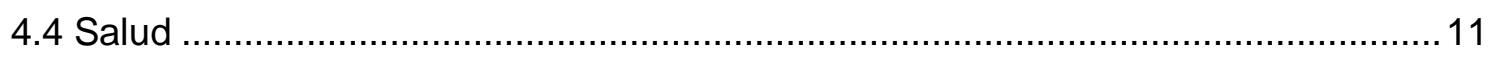

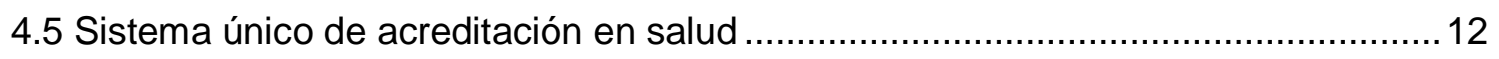

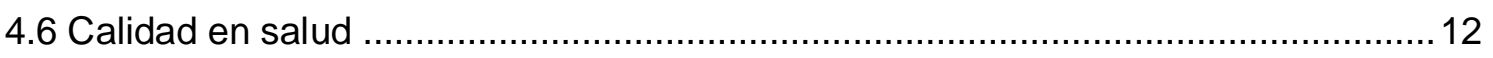

4.7. Configuración de la organización de evaluación externa ......................................13

5. METODOLOGÍA......................................................................................................... 15

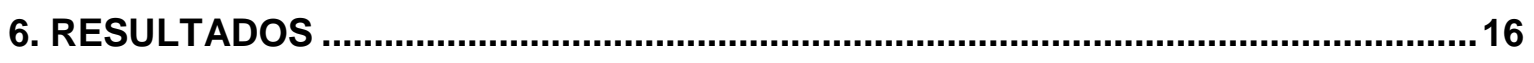

6.1. Organización de evaluación externa ............................................................ 16

6.1.1 Establecimiento de una junta preliminar o comité asesor................................16

6.1.2 Proponer una junta de gobierno y un marco.............................................. 18

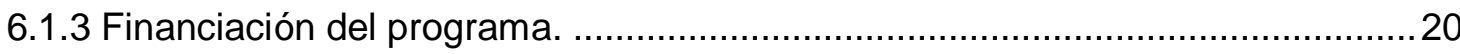

6.1.4 Establecimiento de sistemas de gestión estratégica, operativa y financiera. ....21

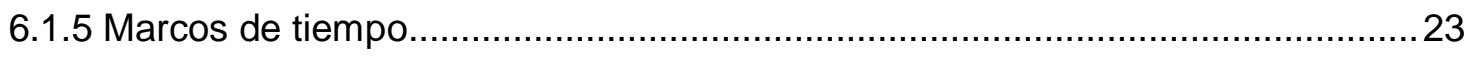

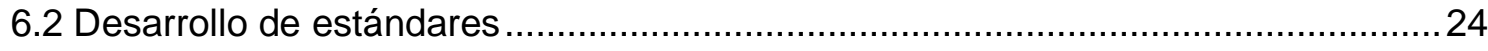

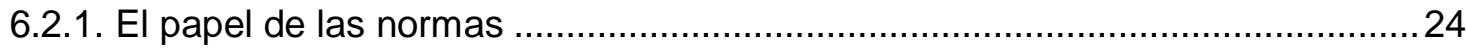

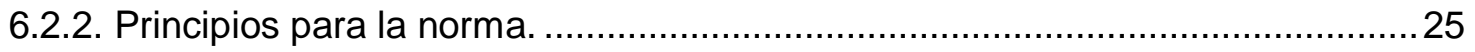

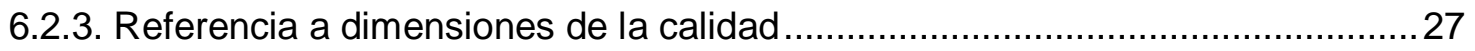

6.2.4. Desarrollo del sistema de medición....................................................................28

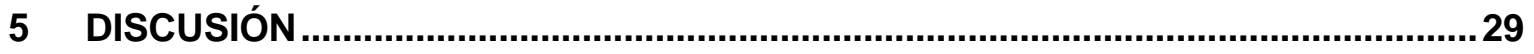

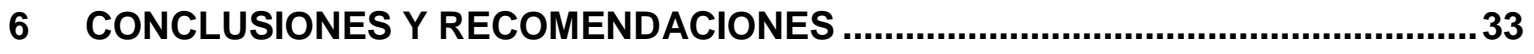

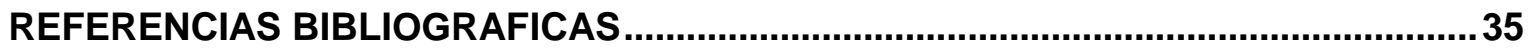




\section{PROBLEMA DE INVESTIGACIÓN}

En la actualidad, la sociedad ha hecho que la salud de vuelva competitivo y dinámico, es así que las empresas e instituciones prestadoras de los servicios de salud se encuentran obligadas a estar en un constante seguimiento de los servicios y/o procesos que se le genera a la sociedad, todo con el fin de crear una calidad de vida óptima de existencia. Con el pasar de tiempo se han ido creando normatividades con el fin de generar medidas que deben ser tomadas e implementadas en las organizaciones para lograr un aumento de calidad de los servicios de salud.

En la actualidad contamos inicialmente con el Sistema de Gestión de la Calidad el cual ayuda a que los servicios que generan las organizaciones sean planeados, controlados y cada vez mejorados con el fin de satisfacer la necesidad de la persona.

Sistema de Calidad del Sector Salud Colombiano (SOGC) definido por el gobierno desde hace doce años, desde el Sistema General de Seguridad Social en Salud, establecido en la Ley 100 de 1993, abarca cuatro componentes los cuales se interrelacionan de manera continua. El ministerio de protección social ajusta de manera periódica y progresiva los estándares de los componentes de SOGC de conformidad con el desarrollo del país, los avances del sector y con los resultados de las evaluaciones adelantadas por las Entidades Departamentales, Distritales de Salud y la Superintendencia Nacional de Salud.

Todo lo anterior abarcando una revisión y un ajuste periódico de los estándares del SOGC los cuales estarán a cargo de la Unidad Sectorial de Normalización (USN), definida por el Ministerio de Protección Social. (1)

Toda organización que solicita ser acreditada, debe de contar con un sistema 
establecido que asegure que los prestadores de servicios de salud y el personal de apoyo sean calificados; que cumplen adecuadamente las necesidades de los pacientes; y que se les ha otorgado los permisos requeridos. Este sistema no sólo debe garantizar que dicho personal sea cuidadosamente seleccionado y que su educación y experiencia sean verificadas a fondo, sino también que su desempeño sea evaluado periódicamente por los superiores y/o sus colegas.

Considerando que las organizaciones que prestan servicios de salud existen para brindar atención a los pacientes, una organización que busca tener nuestra acreditación debe contar con políticas y procedimientos para que aseguren los derechos y responsabilidades de los pacientes. Todo lo anterior con el fin de evitar que estas organizaciones logren ser inhabilitadas para la prestación de los servicios de salud por atentar contra la vida de los pacientes. 


\section{OBJETIVOS}

\subsection{Objetivo general}

Describir los estándares de calidad internacionales con fines de acreditación en el sector salud teniendo como punto de referencia la sociedad internacional para la calidad en el cuidado de la salud (International Society for Quality in Health Care ISQUA)

\subsection{Objetivos específicos}

- Conceptuar los principios, procesos, procedimientos y herramientas que deben usar las organizaciones de salud con fines de acreditación internacionales.

- Detallar los requerimientos de una evaluación externa en el sector salud que demuestren una gestión estratégica, operativa y financiera de una organización. 


\section{JUSTIFICACIÓN}

La Acreditación en Salud es una evaluación específica para el sector salud, que verifica el cumplimiento de estándares de proceso y de resultado de la atención en salud. La acreditación se basa en el cumplimiento de estándares de procesos científicos, administrativos y gerenciales de la atención en salud, enfocados en el usuario y su familia, por parte de las instituciones de salud (IPS y EPS), los cuales se diseñaron teniendo en cuenta el contexto nacional e internacional y aplican a las instituciones como un conjunto integral. (2)

La Acreditación en Salud también es un proceso voluntario, con evaluación por pares, que obedece a unos principios y estándares internacionales, definidos por el organismo acreditador de acreditadores: ISQua o Sociedad Internacional para la Calidad en la Atención en Salud, al I cual ICONTEC está afiliado.

El siglo XXI empezó bien para la salud pública. Cuando los gobiernos de 189 países firmaron la Declaración del Milenio en 2000 y se comprometieron a alcanzar sus objetivos, estaban lanzando el ataque más ambicioso emprendido en la historia contra la miseria humana. El fortalecimiento de los sistemas de salud no era inicialmente una finalidad básica de la mayoría de las iniciativas de salud mundial centradas en una sola enfermedad. Pero ahora sí lo es. (3)

Los líderes mundiales eran optimistas, visionarios, y tenían la determinación de hacer realidad su visión. Surgió un sinfín de iniciativas de salud mundial, muchas de las cuales se propusieron aplicar intervenciones que salvan vidas de forma masiva. (3)

La acreditación representa un reconocimiento a procesos o programas de calidad dentro de una organización teniendo como referencia unos estándares reconocidos, los cuáles garantizan productos o servicios que cumplen con sus 
objetivos, la satisfacción de necesidades de los clientes o usuarios y beneficios administrativos, financieros y reputaciones para la empresa.

Acreditarse significa que el sistema ha logrado superar una serie de indicadores al identificar falencias mediante metodologías internas o externas, que ayudan a corregir las debilidades presentes para el mejoramiento de la organización, incentivando la competitividad, las buenas prácticas y la información oportuna.

En el sector salud la acreditación constituye un aspecto importante para garantizar un servicio de calidad que favorezca el bienestar de todos los usuarios, llevando a cabo este proceso de manera periódica y voluntaria siguiendo una autoevaluación interna y revisión externa de los procesos y resultados, por ello es importante conocer los requisitos y procedimientos que se deben surtir para lograr este reconocimiento en las entidades de salud.

En atención a estos puntos se desarrolla el presente documento acerca de los estándares de acreditación y las metodologías de aplicación en entidades prestadoras de salud, con el objetivo de definir los requisitos que debe cumplir en la práctica una entidad para alcanzar el reconocimiento de la acreditación.

Las Naciones Unidas han desempeñado un papel fundamental en la creación de un consenso internacional sobre las medidas que han de adoptarse para el desarrollo. El sistema de las Naciones Unidas trabaja de diversas maneras para promover los objetivos económicos y sociales. El Programa de las Naciones Unidas para el Desarrollo (PNUD), el principal organismo de las Naciones Unidas que provee subsidios para el desarrollo humano sostenible en todo el mundo, está trabajando activamente en pro de la consecución de las metas de desarrollo establecidas en la Cumbre del Milenio. Los programas del UNICEF se centran en la inmunización, la atención primaria de la salud, la nutrición y la educación básica y comprenden actividades en unos 160 países. (4) 


\section{MARCO TEORICO}

Para el desarrollo del estado del arte en lo referente a procesos investigativos sobre la acreditación en el sector salud con relación al ISQua, se correlacionan los siguientes conceptos:

\subsection{Acreditación}

Acreditación en salud es un proceso voluntario y periódico de autoevaluación interna y revisión externa de los procesos y resultados que garantizan y mejoran la calidad de la atención del cliente en una organización de salud, a través de una serie de estándares óptimos y factibles de alcanzar, previamente conocidos por las entidades evaluadas. Es realizada por personal idóneo y entrenado para tal fin, y su resultado es avalado por la entidad de acreditación autorizada para dicha función (Decreto 1011- Resolución 1445 de 2006).

La acreditación en salud es una metodología de evaluación externa, establecida y reconocida desde hace casi 50 años en Norteamérica, específicamente diseñada para el sector salud, realizada generalmente por una organización no gubernamental, que permite asegurar la entrega de servicios de salud seguros y de alta calidad. Se basa en requisitos de procesos de atención en salud, orientados a resultados y se desarrolla por profesionales de salud que son pares o contrapartes del personal de las entidades que desean acreditarse $y$ de reconocimiento público en el ámbito nacional e internacional, con efectos de mejoramiento de calidad demostrados. Esta evaluación corresponde a un proceso voluntario en el cual se reconoce públicamente a una Institución Prestadora de Servicios de Salud, (IPS), Entidad Promotora de Servicios de Salud del Régimen Contributivo y Régimen subsidiado (EPS), Entidad de Medicina Prepagada (EMP) o Entidad Adaptada, Entidades Territoriales de Salud (ETS), el cumplimiento de estándares superiores de calidad, previo cumplimiento de los requisitos mínimos 
determinados por el Sistema Único de Habilitación.(5)

Los estándares de acreditación han sido diseñados para fomentar el mejoramiento continuo de la calidad y, por ende, se ajustan cada cuatro años. Las organizaciones de salud que deseen mantener su certificado de acreditación, deberán renovarlo igualmente cada cuatro años.

El objetivo del Sistema Único de Acreditación, además de incentivar el manejo de las buenas prácticas, es afianzar la competitividad de las organizaciones de salud y proporcionar información clara a los usuarios, de manera que puedan tomar decisiones basadas en los resultados de la Acreditación y decidir libremente si deben permanecer o trasladarse a otras entidades del sistema que también estén acreditadas.(5)

\subsection{Internacional Society for Quality in Health Care (ISQua)}

Es un organismo sin ánimo de lucro e independiente. Cuenta con miembros en más de setenta países. La ISQua asegura la mejora sostenida de la calidad y la seguridad de los servicios asistenciales. Los servicios que ofrecen ayudan a los profesionales de la sanidad, a alcanzar la excelencia en servicios sanitarios.

La ISQua lanzo su programa internacional de acreditación (IAP) en 1999, se trata de un programa internacional que acredita, a su vez, a los organismos de acreditación. En diciembre de 2009, la ISQua había otorgado la acreditación a 16 organizaciones. (6)

\subsection{Principios de ISQua}

En este sentido el Consejo Directivo de la Sociedad Internacional para la Calidad de la Atención en Salud, ISQua, ha establecido principios guía para el desarrollo de los estándares de atención social y de salud que permitan su evaluación y 
acreditación.

Los principios se basan en las dimensiones de calidad del Instituto de Medicina (OIM), de rendimiento de calidad efectivo, rendimiento organizativo eficiente, seguridad y atención al paciente. Los Principios ISQua también brindan orientación sobre cómo desarrollar y medir estándares. (7)

Los Principios de ISQua cubren todas las funciones de una organización de asistencia social o de asistencia social, desde la gestión, la gestión, la atención al cliente y la calidad son:

1. Desarrollo de estándares: los estándares se planifican, formulan y evalúan a través de un proceso definido y riguroso.

2. Medición de estándares: los estándares permiten una calificación y medición consistentes y transparentes de los logros.

3. Rol organizativo, planificación y desempeño: los estándares evalúan la capacidad y eficiencia de las organizaciones de salud y asistencia social.

4. Seguridad y riesgo: las normas incluyen medidas para gestionar el riesgo y proteger la seguridad de los pacientes /usuarios del servicio, el personal y los visitantes.

5. Enfoque del paciente/servicio al usuario: los estándares se centran en los pacientes / usuarios del servicio y reflejan la continuidad de la atención.

6. Desempeño de calidad: los estándares requieren que los proveedores de servicios monitoreen, evalúen y mejoren regularmente la calidad de los servicios.

\subsection{Salud}

«La salud es un estado de completo bienestar físico, mental y social, y no solamente la ausencia de afecciones o enfermedades». La cita procede del Preámbulo de la Constitución de la Organización Mundial de la Salud, que fue adoptada por la Conferencia Sanitaria Internacional, celebrada en Nueva York del 19 de junio al 22 de julio de 1946, firmada el 22 de julio de 1946 por los 
representantes de 61 Estados (Official Records of the World Health Organization, № 2, p. 100), y entró en vigor el 7 de abril de 1948. La definición no ha sido modificada desde 1948. (8)

\subsection{Sistema único de acreditación en salud}

Es el conjunto de procesos, procedimientos y herramientas de implementación voluntaria y periódica por parte de las instituciones prestadoras de servicios de salud, las Entidades Promotoras de Salud, las Entidades Administradoras de Riesgos Laborales y las Instituciones Prestadoras de Servicios de Salud que presten servicios de salud ocupacional, los cuales están destinados a comprobar el cumplimiento gradual de niveles de calidad superiores a los requisitos mínimos obligatorios, para la atención en salud, bajo la dirección del Estado y la inspección, vigilancia y control de la Superintendencia Nacional de Salud. (9)

\subsection{Calidad en salud}

El Sistema obligatorio de garantía de la calidad en salud para Colombia (SOGC), es producto de un trabajo mancomunado del Ministerio de Salud (actual Ministerio de la Protección Social) y el Consorcio internacional conformado por la Asociación Centro de Gestión Hospitalaria, el Consejo Canadiense de Acreditación de Servicios de Salud y QUALIMED, como resultado del concurso internacional de méritos 06 de 1999 llevado a cabo por el Ministerio con financiación del Banco Interamericano de Desarrollo (BID). El objetivo del proyecto ha sido "Desarrollar y fortalecer el Sistema de garantía de calidad, que integra aspectos de evaluación de la calidad e información al usuario, así como los mecanismos para implementarlo en las entidades aseguradoras y los prestadores, con el fin de garantizar en el mediano plazo una óptima calidad de los servicios a toda la población cubierta por el sistema de seguridad social, y que conlleve una alta satisfacción de los usuarios, al tiempo que impulse el desarrollo de la cultura de la calidad en el sector. (10) 
Es el conjunto de instituciones, normas, requisitos, mecanismos y procesos deliberados y sistemáticos que desarrolla el sector salud para generar, mantener y mejorar la calidad de los servicios de salud en el país. (7)

Existen diferentes métodos para elaborar un programa de evaluación externo, no obstante siempre deben ser fáciles de usar, capaces de cumplir los propósitos para los cuales han sido diseñados, y ser capaces de medir el logro de una manera consistente.

\subsection{Configuración de la organización de evaluación externa}

El ímpetu para establecer una organización de acreditación o certificación puede provenir de una serie de posibles interesados: el Ministerio de Salud, las asociaciones de profesionales de la salud, organizaciones de consumidores, aseguradoras privadas, departamentos universitarios, sociedades de afiliación voluntaria, organizaciones benéficas de servicios de salud u organizaciones de ayuda. La iniciativa puede venir de una compañía o grupo de individuos que ven una oportunidad de mercado, por ejemplo, como asesores de las normas gubernamentales. Si el propósito del programa es claro, no es difícil identificar a quién servirá ya quién afectará. Tradicional, impulsado por la profesión.

Una forma de involucrar a las partes interesadas relevantes que tienen o tendrán interés en el éxito de la nueva organización es a través de la creación de una junta preliminar o un comité asesor para establecer la organización. Esto les permite sentir que tienen un interés en la organización y su trabajo y brindar asesoramiento y experiencia.

Típicamente, los consejos independientes incluyen a los consumidores; representantes de asociaciones profesionales como enfermeras, gerentes y médicos; asociaciones de la industria tales como hospitales o casas de descanso; 
agencias de financiamiento; y los órganos estatutarios. Algunas juntas ahora se designan de acuerdo con las habilidades, la experiencia y la experiencia, en lugar de ser elegidas por organizaciones representativas de partes interesadas debido a los conflictos de interés percibidos que pueden tener los miembros representativos, siendo el proveedor, el consumidor y algunas veces.

La organización de evaluación externa debe establecerse como una entidad legal, o parte de una, con responsabilidades legales claras para todas sus actividades de evaluación externa. Si es parte de un ministerio o agencia gubernamental, esta independencia es particularmente importante.

La organización requiere una visión clara y una misión o propósito y una dirección estratégica para proporcionar la base para la planificación y dirección de la organización y debe guiarse por un conjunto definido de valores que se reflejan en todos los servicios y actividades. También es importante que la organización tenga un conjunto explícito de principios éticos para informar toda la toma de decisiones y un código de conducta que describa los comportamientos esperados de aquellos que trabajan en y/o en nombre de la organización

Las organizaciones de evaluación externa que han tenido éxito en hacer mejoras en las organizaciones de atención médica de los clientes generalmente lo han hecho al estimular la motivación interna y el compromiso con la autoevaluación y el cambio. La experiencia externa de los países es necesaria y debe tenerse en cuenta en los costos iniciales. En la siguiente etapa, cuando se complete el desarrollo inicial y la organización esté lista para ofrecer acreditación o certificación, puede enfrentar otro desafío; cuanto más rápida sea la tasa de captación, más rápido deberá invertir para crear capacidad. La financiación debe ser perfilada para reflejar este crecimiento. 


\section{METODOLOGÍA}

A continuación, se da desarrollo a la metodología aplicada en el presente estado del arte, para identificar los factores y estándares a tener en cuenta para poder optar con el enfoque de acreditación en salud por medio de un programa de acreditación externa de atención médica, en este caso se enfoca en ISQua con el fin de brindar un servicio óptimo para la población.

Los estándares de evaluación externa son herramientas valiosas para examinar la organización e informar al público qué esperar de los proveedores de atención social y de salud, sirviendo además como punto de referencia para que los proveedores y el gobierno puedan medir la calidad de la institución.

El desarrollo de estándares puede variar según la metodología utilizada y el tipo de organización que se tome como referencia, por lo que es recomendable no utilizar los estándares aún esté en la fase de desarrollo, puesto que los resultados pueden no adaptarse a la realidad. La construcción de estándares puede comenzar antes del establecimiento de los sistemas de gobierno y gestión y tardar dos o más años en completarse. 


\section{RESULTADOS}

\subsection{Organización de evaluación externa}

El proceso de establecer una organización de evaluación externa y en las diferentes etapas de este proceso. Este proceso puede ser diferente para cada país o región según la política del gobierno, las partes interesadas involucradas y el tamaño del sector de salud o asistencia social. (7)

Grafica 1: Organización para una evaluación externa

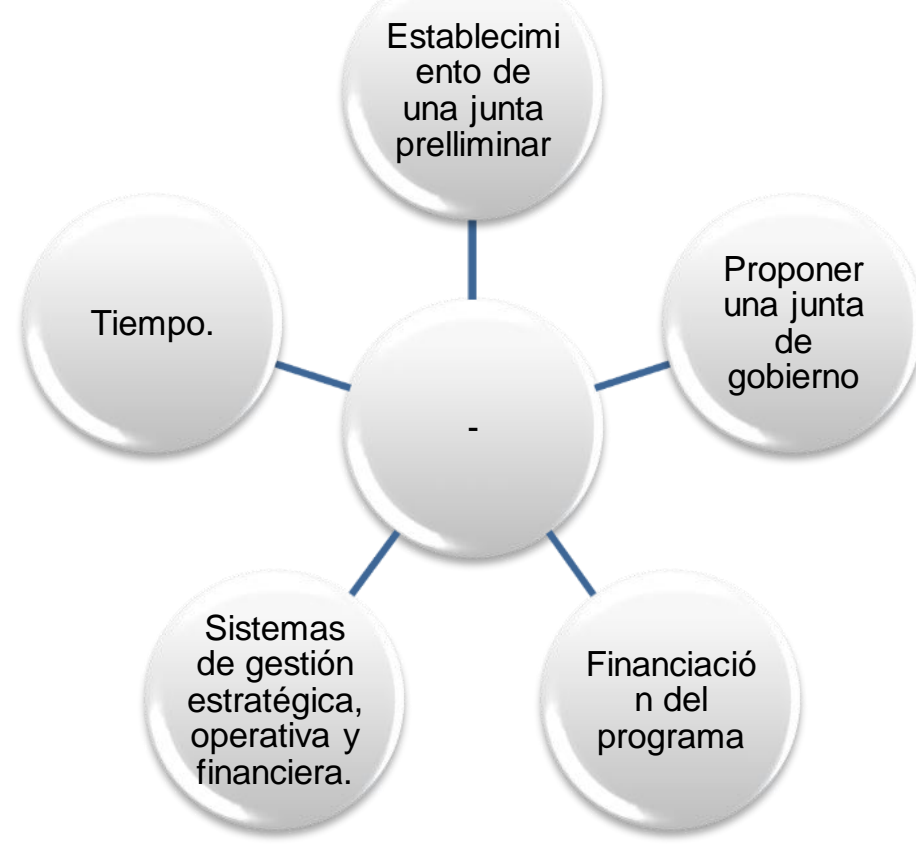

Fuente: Guidance on Designing Healthcare External Evaluation Programmes Including Accreditation. International Accreditation Programme

\subsubsection{Establecimiento de una junta preliminar o comité asesor.}

Los programas han tendido a establecer vínculos con los reguladores y los 
consumidores, por lo que son más responsables y transparentes. Los programas más recientes han sido más influenciados por proveedores comerciales y aseguradores o apoyados activamente por el gobierno. (7)

La junta preliminar o el comité asesor brindarán orientación y orientación sobre los aspectos prácticos del establecimiento del programa de evaluación externa, que incluyen:

- Aclarar la función del programa de evaluación externa en el contexto de otros departamentos y agencias que trabajan en el ámbito de la calidad y la seguridad en el país o jurisdicción.

- Financiación del programa de evaluación externa.

- Marco de gobernanza para la organización de evaluación externa.

- El uso de la asistencia externa para el desarrollo y la entrega del programa de evaluación externa.

La composición de la junta interina o comité asesor será única para cada país dependiendo de la política del gobierno y la gama de partes interesadas que trabajan en el ámbito de la calidad y la seguridad. Algunos miembros de esta junta o comité pueden formar la base para la junta de gobierno en la organización establecida. (7)

Tabla 1: Composición potencial de una junta preliminar o comité asesor

\begin{tabular}{|c|c|}
\hline $\begin{array}{l}\text { Grupo de partes } \\
\text { interesadas }\end{array}$ & Ejemplos de representantes \\
\hline Gobierno & $\begin{array}{l}\text { Ministerio de Salud y/u otros departamentos relacionados, por } \\
\text { ejemplo. Financiar. } \\
\text { Gobierno local, por ejemplo: Municipio, cantón. }\end{array}$ \\
\hline $\begin{array}{l}\text { Grupos de } \\
\text { consumidores }\end{array}$ & $\begin{array}{l}\text { Consejo/asociación nacional de consumidores reconocida u } \\
\text { organización de defensa }\end{array}$ \\
\hline
\end{tabular}




\begin{tabular}{|c|c|}
\hline $\begin{array}{l}\text { de evaluación } \\
\text { externa }\end{array}$ & $\begin{array}{l}\text { que trabajan en el ámbito de la calidad y la seguridad en el } \\
\text { país o jurisdicción, por ejemplo. organismos estatutarios } \\
\text { responsables de áreas como salud y seguridad, radiación, } \\
\text { dispositivos médicos, medicamentos, inspecciones } \\
\text { reglamentarias, agencias de certificación }\end{array}$ \\
\hline $\begin{array}{l}\text { Proveedores de } \\
\text { servicios }\end{array}$ & $\begin{array}{l}\text { Proveedores públicos y privados en el país o región, p. Ej. } \\
\text { organismos representativos nacionales, como la asociación de } \\
\text { hospitales nacionales o la asociación / foro nacional de } \\
\text { proveedores de servicios de discapacidad }\end{array}$ \\
\hline $\begin{array}{l}\text { Organismos } \\
\text { profesionales }\end{array}$ & $\begin{array}{l}\text { Organismos independientes responsables de la concesión de } \\
\text { licencias o el registro de profesionales de la salud y de la } \\
\text { asistencia social o la supervisión de la formación, como } \\
\text { academias médicas o consejos. }\end{array}$ \\
\hline Academia & $\begin{array}{l}\text { Universidades o colegios que ofrecen programas de educación } \\
\text { y capacitación para profesionales de la salud y asistencia } \\
\text { social. }\end{array}$ \\
\hline $\begin{array}{l}\text { Agencias } \\
\text { técnicas }\end{array}$ & $\begin{array}{l}\text { Agencias nacionales con un rol específico, por ejemplo: } \\
\text { Evaluaciones de tecnologías sanitarias, guías clínicas y vías, } \\
\text { seguridad del paciente / consumidor. }\end{array}$ \\
\hline Independiente & $\begin{array}{l}\text { Expertos independientes, organizaciones vecinas de } \\
\text { evaluación externa, iniciativas internacionales de evaluación } \\
\text { externa. }\end{array}$ \\
\hline
\end{tabular}

Fuente: Guidance on Designing Healthcare External Evaluation Programmes Including Accreditation. International Accreditation Programme

\subsubsection{Proponer una junta de gobierno y un marco.}

Una de las primeras tareas de la junta será desarrollar un borrador de marco de gobernabilidad para la organización o programa de evaluación externa, con una constitución formal, una junta de gobernanza y políticas y procedimientos preliminares. Para la credibilidad y en línea con las mejores prácticas, se debe 
hacer un compromiso de que la organización se establecerá de acuerdo con las Pautas y Estándares de ISQua para la Evaluación Externa.

Grafica 2: Requisistos de la junta de gobierno

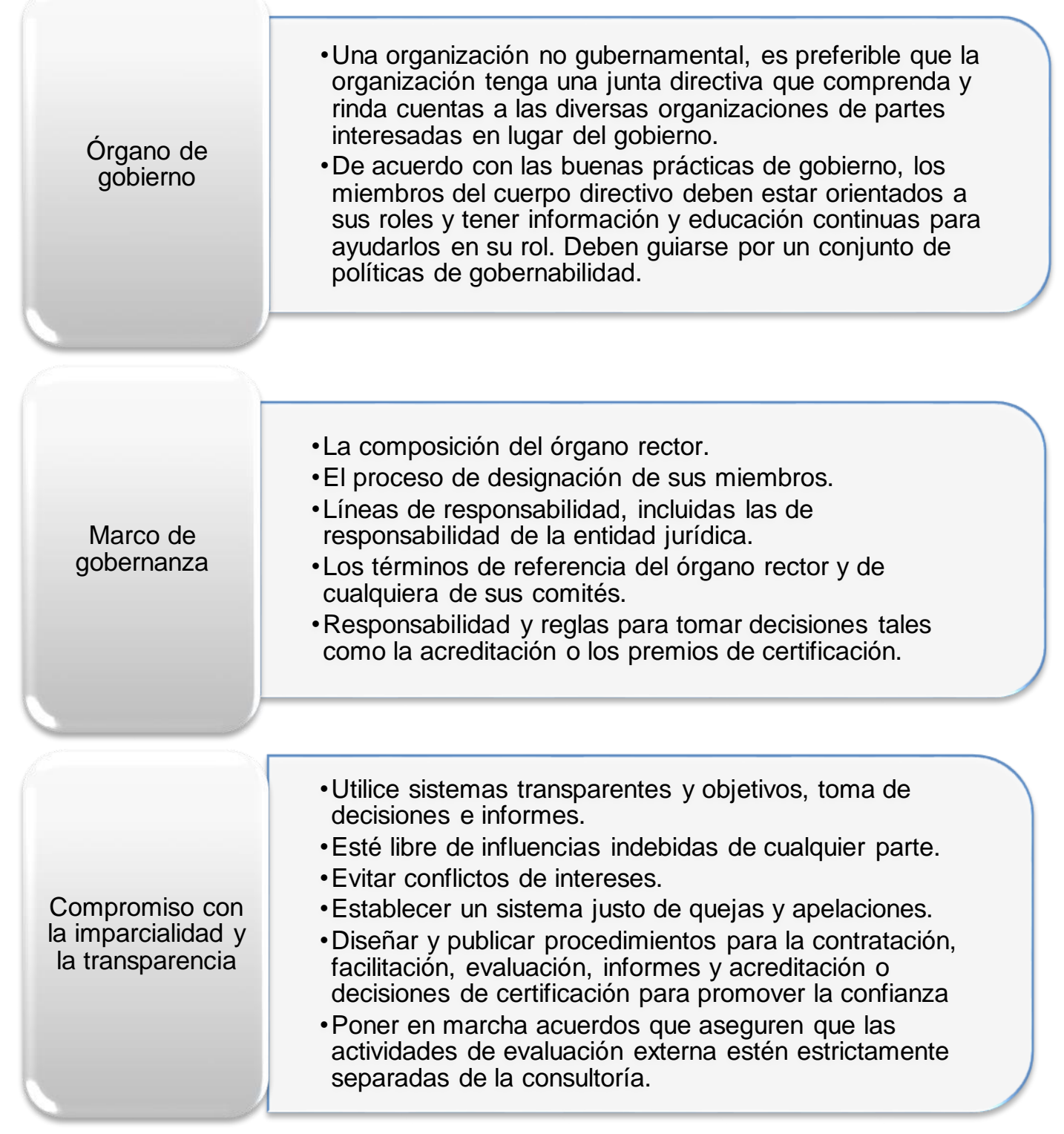

Fuente: Guidance on Designing Healthcare External Evaluation Programmes Including Accreditation. International Accreditation Programme 


\subsubsection{Financiación del programa.}

La mayoría de las nuevas organizaciones de evaluación externa requieren al menos dos años para establecer su organización y/o programa, más tiempo antes de que sean sostenibles y aún más antes de que se autofinancien. El apoyo político y financiero generalmente necesita ser consistente más allá del término en el cargo de la mayoría de los ministros de salud y muchos gobiernos. Se requerirá financiamiento externo del gobierno, aseguradores de salud, organizaciones de ayuda u otros socios, recursos que serán destinados para lo siguiente:

Grafica 3: Designación de financiamiento

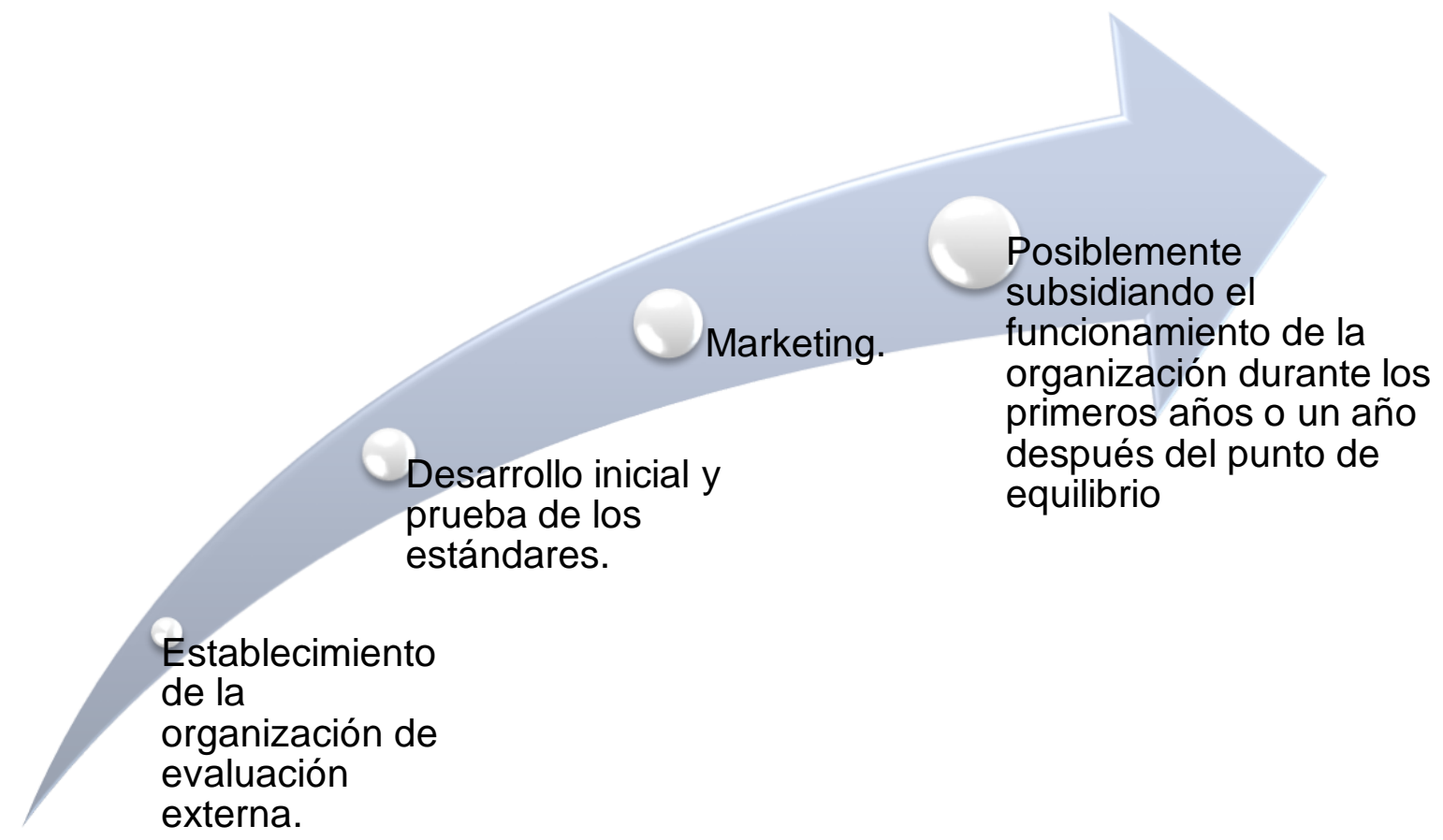

Fuente: Guidance on Designing Healthcare External Evaluation Programmes Including Accreditation. International Accreditation Programme

Para la mayoría de las organizaciones, la cantidad de organizaciones de atención social o de salud de clientes potenciales será un determinante clave de los costos del programa, al igual que otros factores, como si el programa: 
Grafica 4: Determinantes claves de los costos

Es un programa nacional único, regional o sectorial específico.
Se limita inicialmente a un enfoque prioritario, por ejemplo, hogares de ancianos, o para todo el sistema de salud.

Está enfocado al desarrollo, requiere capacitación y educación de los clientes.
Desarrolla sus propios estándares.
Está complementando

o reemplazando las evaluaciones externas existentes.
Emplea expertos especializados.

Fuente: Guidance on Designing Healthcare External Evaluation Programmes Including Accreditation. International Accreditation Programme

\subsubsection{Establecimiento de sistemas de gestión estratégica, operativa y financiera.}

Una vez que se ha establecido la junta de gobierno y se ha desarrollado el marco de gobierno, el siguiente paso es dotar de personal a la organización de evaluación externa y desarrollar los sistemas de gestión.

\section{Dotación de personal de la organización}

En organizaciones más grandes, el personal puede estar estructurado en unidades funcionales tales como:

- Planificación y gestión de encuestas.

- Selección y desarrollo de topógrafos

- Investigación, desarrollo y revisión de estándares

- Educación y desarrollo del usuario

- Personal de soporte técnico - financiera, recursos humanos, gestión de la 
información.

- Administración

Desarrollo del sistema de sostenibilidad financiera

El presupuesto inicial es desafiante y depende de cuánta financiación se reciba para el desarrollo o cuánto de los costos de configuración deben incluirse en el presupuesto. Por lo general, es necesario prever la asistencia externa y la experiencia. Algunas organizaciones consideran la facilitación guiada y / o la capacitación sobre los estándares y el proceso de la encuesta como parte integral del proceso de desarrollo

\section{Establecimiento de sistemas de información}

Los sistemas de información internos son esenciales para la planificación, las operaciones y las finanzas, pero también deben tener la capacidad de recopilar, agregar y comparar datos a lo largo del tiempo dentro y entre las organizaciones participantes y las normas

- Datos de cumplimiento con el cumplimiento de criterios o estándares individuales

- Perfiles de organizaciones participantes

- Cálculo de puntajes estándar, puntajes de función y puntaje general para cada organización

- Resultados agregados para la comparación en el tiempo, la función y el lugar.

- Perfiles de los topógrafos individuales y su participación.

- Programación y gestión de encuestas.

- Impacto global del programa.

\section{Abordar la gestión de riesgos y la mejora del rendimiento}

La organización de evaluación externa debe modelar el enfoque de seguridad y calidad que espera de sus organizaciones clientes. Un robusto marco de gestión de riesgos que Identifica y gestiona los riesgos y promueve la seguridad que deben implementarse 
Prestación de servicios educativos.

- Inducción y desarrollo del personal.

- Orientación y educación continua de los miembros de la junta directiva.

- Capacitación inicial y continua de los topógrafos.

- Preparación general de las organizaciones participantes y su personal como componente básico de su participación.

- Métodos específicos de mejora de la calidad interna requeridos para cumplir con los estándares de evaluación externa, como el control de infecciones, la gestión de riesgos, la medición del rendimiento y las encuestas de pacientes / clientes. Estos son generalmente adicionales a los servicios cubiertos por tarifas y se cobran por separado

- Programas de mejora de la calidad para el sector salud o asistencia social en general.

\subsubsection{Marcos de tiempo}

El recurso más comúnmente subestimado es el tiempo necesario para planificar, diseñar, construir y entregar una nueva organización de evaluación externa sostenible. El ritmo al que se puede hacer esto está limitado en gran medida por factores que están fuera del control de la organización, en particular por la cultura y las actitudes prevalecientes hacia el liderazgo, la innovación, la mejora, el trabajo en equipo y la transparencia.

Grafica 5: Etapas de desarrollo en la practica 


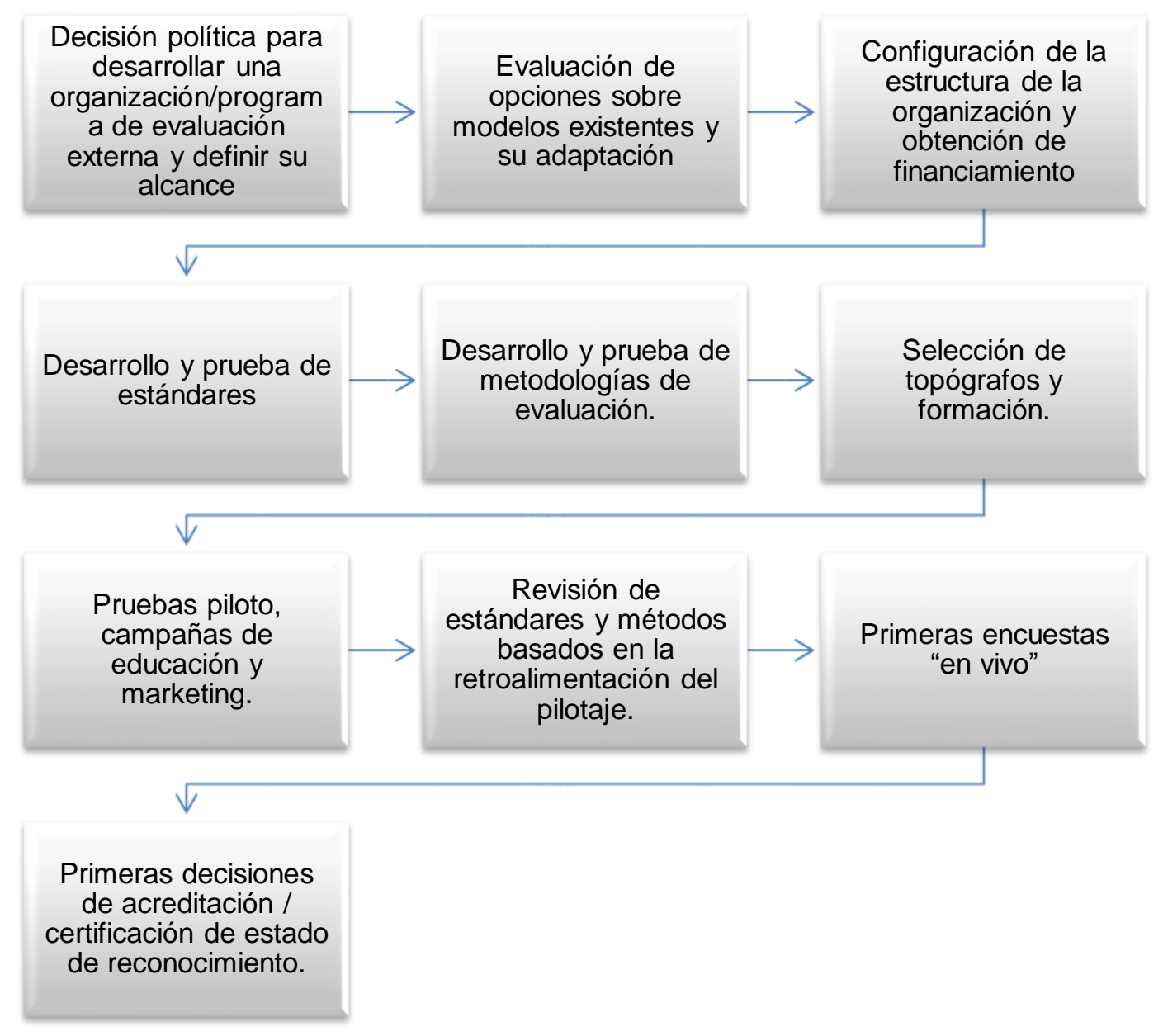

Fuente: Guidance on Designing Healthcare External Evaluation Programmes Including Accreditation. International Accreditation Programme

\subsection{Desarrollo de estándares}

El desarrollo de estándares a menudo puede comenzar antes del establecimiento de los sistemas de gobierno y gestión en la organización de evaluación externa y puede tardar dos o más años en completarse. Los financiadores pueden querer saber la forma y el contenido de los estándares antes de comprometerse a financiar a la organización

\subsubsection{El papel de las normas}

Los estándares de una organización de evaluación externa deben reflejar su 
propósito y cubrir las funciones y procesos clave de los sectores de atención médica o asistencia social que se están evaluando. De manera similar, si los estándares son propiedad o están obligados por el gobierno, deben reflejar el propósito para el cual el gobierno los pretende. Deben reflejar requisitos legislativos, seguridad y buenas prácticas.

Los estándares también deben ser realistas y reflejar la disponibilidad de recursos, especialmente en los países en desarrollo donde las limitaciones de recursos pueden afectar significativamente la capacidad de una organización de atención médica para lograr un rendimiento óptimo. La evaluación externa puede ser principalmente un vehículo para hacer un balance y desarrollar una mayor igualdad de estructura y acceso donde el sistema de salud

\subsubsection{Principios para la norma.}

ISQua se ha centrado en abordar esta brecha mediante el desarrollo de principios que guíen el desarrollo de los estándares de atención social y de salud y permitan su evaluación y acreditación.

Los pasos para desarrollar estándares en línea con los Principios de ISQua incluyen:

Grafica 6: Pasos para desarrollar estándares en línea 


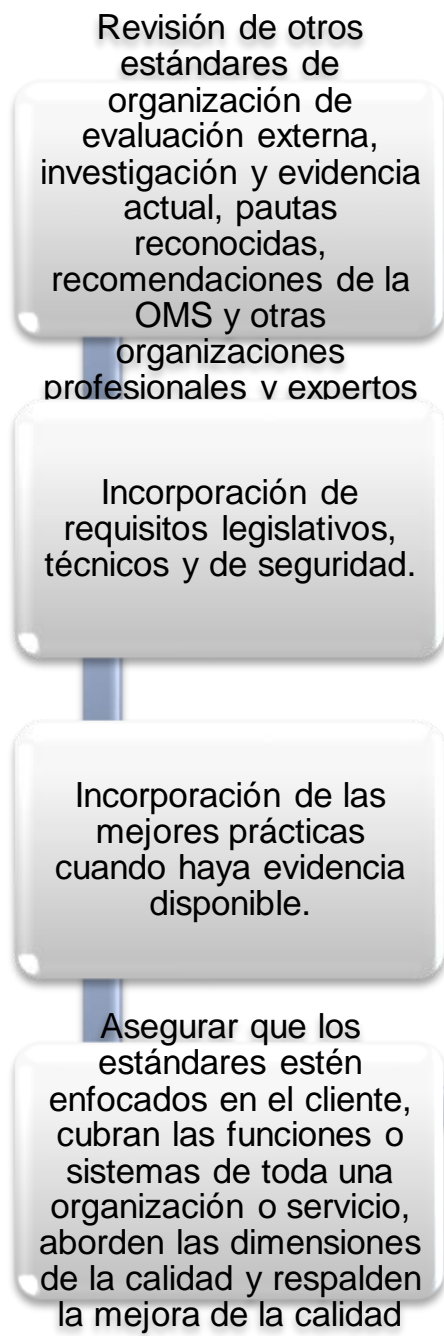

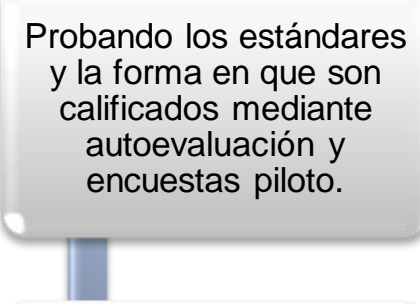

Desarrollar el sistema de calificación para medir el cumplimiento con/contra las normas.

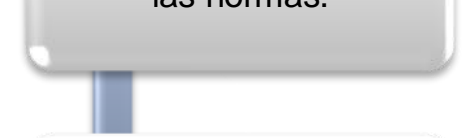

Involucrar a las partes interesadas en los comités de desarrollo de normas y grupos de trabajo

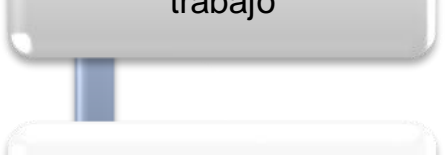

Consultando grupos de partes interesadas, incluyendo grupos de consumidores.
Uso de los comentarios de las pruebas para mejorar los estándares y el sistema de calificación.

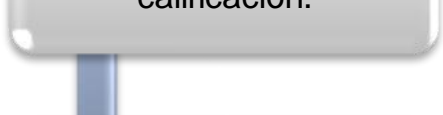

Desarrollar pautas para ayudar a los usuarios a interpretar y aplicar los estándares.

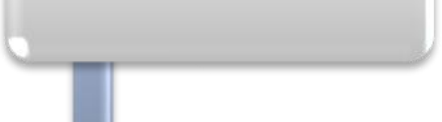

Asegurar que las normas sean aprobadas por el organismo rector de la evaluación externa.

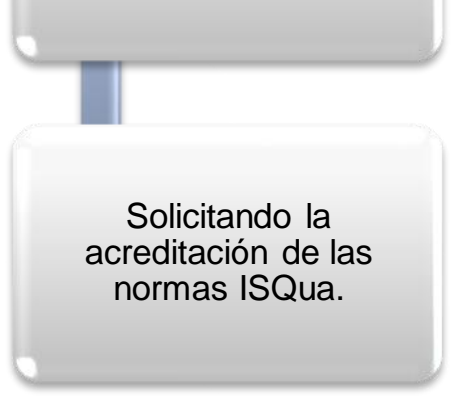

Fuente: Guidance on Designing Healthcare External Evaluation Programmes Including Accreditation. International Accreditation Programme

Este proceso de desarrollo puede tardar dos años o más si los estándares se están desarrollando completamente. Con el entorno sanitario que cambia rápidamente, 12 meses sería un plazo adecuado para que las organizaciones 
adapten los estándares de otras organizaciones

\subsubsection{Referencia a dimensiones de la calidad}

Las normas pueden agruparse en torno a las dimensiones de calidad para demostrar su relación con la calidad.

Grafica 7: Dimensiones de la calidad

\section{Seguro S}

Oportuno T

Eficiente $\mathbf{E}$

Equitativa $\mathbf{E}$

Efectiva $\mathbf{E}$

Centrada

en el

paciente $\mathbf{P}$

Fuente: Guidance on Designing Healthcare External Evaluation Programmes Including Accreditation. International Accreditation Programme

Muchos conjuntos de estándares etiquetan algunos criterios como básicos $u$ obligatorios, generalmente basados en la seguridad y el riesgo. Por lo general, se requiere que los criterios centrales se cumplan o que se cumpla una proporción definida de ellos, por ejemplo. $80 \%$. Estos criterios básicos pueden utilizarse para 
fines de licencia o regulación.

\subsubsection{Desarrollo del sistema de medición.}

La escala de calificación debe reflejar el propósito de los estándares, ser transparente y permitir a los usuarios calificar y medir estándares, criterios o elementos de manera consistente. Una escala de sí / no es buena para determinar el cumplimiento o el incumplimiento de un criterio o norma, especialmente para medir elementos estructurales, por lo que su uso debe reflejar la naturaleza de las normas. Deja menos margen para recomendaciones de mejora cuando un criterio se cumple principalmente, pero faltan algunos elementos.

Las escalas de calificación tipo Likert son particularmente adecuadas para estándares con un fuerte enfoque de mejora de la calidad, por ejemplo, Escalas de 3, 5 o 7 puntos, a menudo con descripciones para cada punto o algunos de los puntos. Estas descripciones pueden relacionarse con principios como el cumplimiento, la coherencia, la evidencia y la implementación.

Al igual que el resto de los estándares, la escala de calificación debe desarrollarse en consulta con las partes interesadas y la satisfacción de los usuarios evaluados regularmente. Al igual que con los estándares en sí, la escala de calificación debe probarse y probarse antes de su uso para garantizar que sea confiable y pueda producir resultados consistentes y justos. 


\section{DISCUSIÓN}

En Colombia y en general en el mundo, se han desarrollado muy pocos estudios que realmente midan el impacto de los procesos y resultados administrativos como elementos componentes de la calidad en salud, máxime si se trata de procesos apalancados en sistemas y estrategias, tales como la acreditación de los servicios. Estudios de gran magnitud, de los cuales el más reciente ejemplo es el IBEAS, tienen una clara orientación hacia la medición de indicadores relacionados con resultados asistenciales, tales como la gestión de eventos adversos y la seguridad del paciente.(11)

Existe varias organizaciones de evaluación externa tanto nacional como internacional, en este caso, nos enfocamos en la Sociedad Internacional para la Calidad en la Atención de la Salud (ISQUA), en donde mediante la guía "Guidance on Designing Healthcare External Evaluation Programmes Including Accreditation" de ISQua, en donde se establecen las principales y primordiales procesos que se deben llevar a cabo, las condiciones a tener en cuenta para poder prestar un servicio de calidad en salud.

La evaluación externa basada en el establecimiento de una organización encargada de llevar múltiples procesos de importancia para la acreditación a nivel internacional se encuentran basados en el establecimiento de una junta preliminar, una junta de gobierno, el financiamiento del programa, los sistemas a utilizar en la gestión de estrategias y finalmente el periodo de tiempo el cual va a tomar y el periodo de durabilidad.

La participación pública como intercambio de información también exige compartir la autoridad. Muchas organizaciones de evaluación externa tienen representantes de pacientes y el público en su estructura de gobierno para garantizar su participación en el desarrollo de políticas y estándares y para garantizar que se 
sigan los procedimientos acordados a lo largo del proceso de evaluación externa.(7)

Los estándares a desarrollar van determinados por los roles en los que se encuentran establecidas las diferentes normas nacionales e internaciones haciendo así caso oportuno y guiándose siempre por la legislación vigente en la actualidad. La organización ISQua centrada en la atención de salud y la atención social, permite que sea más factible una evaluación dirigida a la acreditación, siguiendo los pasos establecidos para tal fin.

Uno de los principales costos potenciales para una organización de evaluación externa será la fuerza de trabajo del inspector y, en particular, si son remunerados o voluntarios. Tradicionalmente, las organizaciones de acreditación han confiado en las instituciones acreditadas participantes para proporcionar o prestar personal para que trabajen como topógrafos y promuevan el concepto de revisión por pares. (7)

La mayoría de los programas de evaluación externa proporcionan una variedad de educación y capacitación como un componente esencial de sus servicios. Los servicios de educación deben diseñarse e implementarse sistemáticamente para cumplir con los estándares de calidad y las necesidades de los clientes (7)

Las dimensiones de la calidad pueden agruparse para demostrar su relación con la calidad, como se mencionaron seis dimensiones de la calidad las cuales hacen referencia a seguridad, oportunidad, eficiencia, equitativa, efectiva y centrada en el paciente. Al incluir las dimensiones de la calidad asociadas al servicio y procesos de la organización se establecen parámetros definidos y claros como parte de la atención diaria.

Las organizaciones pueden catalogar los estándares mediante conceptos subjetivos de importancia, según criterios como básicos u obligatorios, 
habitualmente apoyados en la seguridad y el riesgo. Los criterios normalmente poseen una escala que mide el nivel de cumplimiento, la cual pueden utilizarse como una medida de regulación del cumplimiento y de satisfacción.

La escala de evaluación se debe diseñar para que exprese de manera clara y precisa el objetivo del estándar, dando la posibilidad de que los usuarios puedan calificar de forma práctica el servicio. La escala puede ser del tipo sí / no reflejando el cumplimiento del criterio o norma. También se pueden utilizar escalas de calificación tipo Likert que trabajan con puntuaciones, las cuales son especiales para estándares con enfoque de mejora de la calidad. Las puntuaciones pueden asociarse con principios como el cumplimiento, la coherencia, la evidencia y la implementación.

Es necesario que además de poder determinar si los estándares se cumplen de manera individual también tener la posibilidad de evaluar el cumplimiento general, ya que ello constituye la base para lograr la acreditación o certificación. La mejor práctica es determinar el logro general de los estándares basándose en una fórmula que incluya el nivel de logro o cumplimiento de los elementos medibles de los estándares, el riesgo y otros elementos de los estándares, como los criterios básicos o los criterios de alta prioridad.

Los estándares y la escala de calificación deben desarrollarse en consulta con las partes interesadas y la satisfacción de los usuarios evaluados regularmente. Estos deben probarse varias veces antes de ser aprobados para garantizar que son confiables y logran resultados fehacientes.

En cuanto al desarrollo de metodologías de evaluación existen varios factores que deben considerarse, tal como son la selección, capacitación y evaluación de los encuestadores; el desarrollo del proceso de gestión de la encuesta; y el establecimiento de procesos para determinar el estado de acreditación o certificación. 
En este proceso es factible llevar a cabo una encuesta relacionada con los estándares, la cual puede ser a través de una revisión de escritorio o una encuesta en el sitio. Las revisiones de escritorio pueden ser adecuadas para algunas especialidades, como la imagenología diagnóstica o vías clínicas como la atención al ictus. Para las organizaciones se recomienda una encuesta en el sitio, que puede ser planificada o no anunciada.

Las organizaciones de acreditación usan el término "topógrafos", mientras que las organizaciones de certificación usan los términos "asesores" o "auditores". Los organismos reguladores utilizan el término "inspectores". Los encuestadores son muy importantes pues garantizan la credibilidad, objetividad y sostenibilidad de la organización. Generalmente son considerados revisores pares, que entienden el trabajo que hacen sus compañeros, pero su función es evaluar los procesos y sistemas en lugar del desempeño de sus pares.

Así mismo son importantes para el proceso de evaluación los auditores, quienes generalmente están certificados en esa función, teniendo la capacidad de auditar o evaluar empresas del sector salud sin que necesariamente sea un profesional de la salud.

Es común que las organizaciones encarguen a instituciones acreditadas que proporcionen o presten personal para que trabajen como encuestadores. Esto tiene la ventaja de reducir los costos de la encuesta, manteniendo la aceptabilidad e independencia de la revisión por pares, y compartir ampliamente la experiencia y el conocimiento de la acreditación en todo el sistema de salud. Sin embargo, se supone que hay son personal con suficiente experiencia que pueden y están dispuestos a ser apoyados por sus empleadores para recibir capacitación como topógrafos sin crear un conflicto de intereses. 


\section{CONCLUSIONES Y RECOMENDACIONES}

La acreditación en salud es una metodología de evaluación externa con parámetros especialmente establecidos para este sector, que propone una serie de estándares óptimos y factibles para alcanzar mayores niveles de eficiencia y calidad en los servicios que se prestan. La acreditación ha sido diseñada para promover el mejoramiento constante de las entidades de salud mediante el examen periódico de sus procesos y servicios, buscando garantizar que la red de atención cubra las necesidades de los usuarios y se consiga un adecuado respaldo a las políticas de salud y sanidad pública.

A nivel nacional existe el Sistema Único de Acreditación en Salud, que cubre a las instituciones prestadoras de servicios de salud, las Entidades Promotoras de Salud, las Entidades Administradoras de Riesgos Laborales y las Instituciones Prestadoras de Servicios de Salud que presten servicios de salud ocupacional, los cuales están sujetos a lograr unos requisitos mínimos obligatorios, para la atención en salud, según las normas establecidas por el Estado y la inspección, vigilancia y control de la Superintendencia Nacional de Salud.

La acreditación no solo beneficia a las entidades del sistema de salud sino en general a todos los colombianos al lograr una mejora continua en la calidad de los servicios y en la atención, por ello es importante lograr conocer la metodología, requisitos y sistemas de evaluación para alcanzar el otorgamiento de la acreditación.

Tal como se pudo observar a través del presente documento, la gestión de mejoramiento que representa la acreditación en el sector salud exige el cumplimiento gradual de los estándares establecidos, para lo cual es necesario seguir un conjunto de procedimiento enfocados a la implementación de indicadores y consecución de objetivos de calidad. 
A partir de la autoevaluación el proceso de acreditación influirá de manera positiva la configuración de la organización transformado la cultura organizacional y la ética, debido al fomento de las buenas prácticas, el aprendizaje organizacional y el logro de conocimientos, aplicados a estrategias de desarrollo que contribuyen a generar una mayor la responsabilidad social y el bienestar de la población. 


\section{REFERENCIAS BIBLIOGRAFICAS}

1. Acreditas global. ¿Cómo contribuye la Acreditación a la mejorar y elevar la Calidad de la Atención en Salud?. [Online]; 2014 [cited 2018/11/27. Available from http://acreditasglobal.org/mejoraryelevar/

2. Instituto Colombiano de Normas Técnicas y Certificación, ICONTEC. Evaluación de la conformidad. [Online]; 2016 [cited 2018/11/27. Available from http://www.icontec.org/Ser/EvCon/Paginas/Prf.aspx

3. Naciones unidas, UN. Visión general. [Online]; 2018 [cited 2018/11/27. Available from http://www.un.org/es/development/other/overview.shtml

4. Organización mundial de la salud, OMS. La creciente importancia de la salud mundial. [Online]; 2018 [cited 2018/11/27. Available from http://www.who.int/dg/speeches/2011/globalhealth_20110613/es/

5. Instituto Colombiano de Normas Técnicas y Certificación, ICONTEC. Que es acreditación en salud. [Online]; 2016 [cited 2018/11/13. Available from http://www.acreditacionensalud.org.co/sua/Paginas/AcrSal.aspx

6. Agencia de salud y servicios sociales de Montreal. Guía para el fomento de puestos de trabajo saludables en los servicios de salud. Documental universitaria. Girona, Diciembre de 2012.

7. Fortune, T; Connor, E; Donaldson, B. Guidance on Designing Healthcare External Evaluation Programmes Including Accreditation. International Accreditation Programme (IAP). 2015

8. Organización mundial de la salud, OMS. Salud según la OMS. [Online]; 2018 [cited 2018/11/13. Available from https://www.who.int/suggestions/faq/es/

9. Ministerios de salud y protección social, MinSalud. ABC SOBRE EL SISTEMA ÚNICO DE ACREDITACIÓN EN SALUD. [Online]; 2018 [cited 2018/11/13. Available from https://www.minsalud.gov.co/sites/rid/Lists/BibliotecaDigital/RIDE/DE/CA/abcsuas.pdf 
10. Kerguelén, C. Calidad en salud en Colombia. Editorial Scripto. 2008.

11. Arenas, C; Tamayo, C. Indicadores administrativos y financieros antes y después de la acreditación en las instituciones prestadoras de servicios de salud en Colombia. Revista CES MEDICINA Volumen 24 No.2 Julio - Diciembre, 2010. 